\title{
Characterization of Strange Attractors of Lorenz Model of General Circulation of the Atmosphere
}

\author{
C. MASOLLER," A.C. SICARDI SCHIFINO* and L. ROMANELLI"* \\ * Instituto de Física, Facultad de Ciencias, Tristán Narvaja 1674 Montevideo, Uruguay \\ and Instituto de Fisica, Facultad de Ingeniería Ilerrera y Reissig 565 Montevideo, Uruguay. \\ ** Departamento de Física, F.C.E. y N., U.U.A, Ciudad Uuiversitaria (1428) Buenos Aires, \\ Argentina.
}

\begin{abstract}
The dynamics of the Lorenz model of general circulation of the atmosphere is investigated. The attractors found are characlerized by calculating their Fourier spiectra, Lyapunov exponents and dimensions. In addition, the self similarity of the attractors is studied with the aid of a Poincare map. A series of one-dimensional maps derived from the Poincaré section illustrates the structural changes of the attractors as a function of parameters variations.
\end{abstract}

\section{INTRODUCTION}

The search for chaotic dynamics has become a fascinating subject in many scientific disciplines, and a great deal of interest has been focused on the relevance of strange attractors in modeling real systerns. Numerical and theoretical evidence for strange attractors has beeu found in many model equations, and several techniques to analyze chaotic dynanics have been developed $[1,2]$. In order to compare model calculations with experimental data, the characterization of the geometrical and statistical properties of the strange attractors is required. In the present paper we present such study of chaotic attractors of the Lorenz model, a model of considerable importance in the fields of meteorology, fluid dynamics and atmospheric sciences.

In Refs. [3,4] Lorenz introduced a modified version of his fanous !.orenz equations [5], a low-order geostrophic baroclinic model capable of representing the general circulation of the atmosphare. In previous studies we have shown that this model exhibits very rich dynamics [6,7]. An immense variety of bifurcation sequences were found, and coexistence of several attractors was reported. In the present contribution we characterize qualitatively these strange attractors, and investigate period-doubling cascades leading to chaos.

Lorenz model of general circulation of the atmosphere consists of the following nonlinear ordinary differcutial equations $[3,4]$

$$
\begin{gathered}
\frac{d X}{d t}=-Y^{2}-Z^{2}-a X+a F \\
\frac{d Y}{d t}=X Y-b X Z-Y+G \\
\frac{d Z}{d t}=b X Y+X Z-Z
\end{gathered}
$$


where the variable $X$ represents the strength of a large-scale westerly-wind current, while $Y$ and $Z$ are the strengths of the cosine and sine phases of a chain of superposed waves. The parameter $F$ represents the external-heating contrast, and $C$ represents the heating contrast between oceans and continents. For $a=1 / 4, b=4, G=1$ and different intensities of the external thermal forcing $F$, these equations may have one or more stable solutions, which can be steady-states, periodic solutions, or aperiodic solutions.

We will now describe briefly the changes that occur in the qualitative nature of the attractors as the parameter $F$ is varied $[6,7]$. For $F<1.18$ the equations possess one stable steady-state solution (fixed point 1), for $1.18<F<1.27$ two stable steady state solutions (fixed points 1 and 2 ), and for $1.27<F<4.31$ one stable steady state (fixed point 1) and one periodic solution (weak limit cycle). The weak cycle period-doubles at $F=6.25$ and becomes unstable at $F=7.85$. In the region $4.31<F<7.85$ the model presents two different attractors (the weak cycle and the strong attractor) and the diagram of solutions becomes extremely complicated. There are regions of the parameter $F$ in which the strong attractor is periodic, regions in which it is aperiodic, and regions in which it does not exist, or is barely stable and only the weak limit cycle exists. In the turbulent region $7.85<F<8.0$ the weak cycle is unstable and the equations have only aperiodic solutions.

We will study in detail three of the strong chaotic attractors found in [7] attractor $B(F=4.56)$, attractor $N(F=5.198)$ and the strongly chiotic altractor $(F=8.0)$. While the transition sceriarios of attractors $B$ and $N$ are period-doubling cascades, the strongly chaotic attractor is born when a long chaotic transient becomes stable after the subcritical llopf bifurcation of the weak limit cycle. The qualitative nature of these attractors is studied with the aid of phase portrait and power spectrum analysis $[1,2,8]$. We find that attractors $B$ and $N$ look like "noise limit cycles". In contrast, the dynamics in the last one is highly chaotic. In addition, the Poincare section of the strongly chaotic attractor presents the self-similar structure characteristic of strange attractor: [9] and is considerably more complicated than the Poincaré sections of attractors $B$ and $N$. The spectrum of Lyapunov exponents [10-12] and several dimensions [13-16] are employed for dynamical and geometrical characterization. We show that these attractors have one positive Lyapunov exponent and fractal dimension. In addition, the period doubling cascades found in [7] are studied in detail. We show that these routes are in perfect correspondence to the Peigenbaum sequence $[17,1 ;]$. A series of one dimensional maps is derived from the Poincare section that explains this behavior.

The paper is organized as follows. In section 2 the phase portrait and power spectrum of the attractors are studied, and their Poincaré sections are presented in section 3 . In section 4 the period-doubling routes to chaos are analyzed. Section 5 is concerned with the dynamical and geometrical characterization with the spectrum of Lyapunov exponents and dimensions. Finally, section 6 presents the discussion of the results.

\section{VISUALIZATION OF THE DYNAMICS}

In order to study the qualitative nature of attractors $B, N$ and the strongly chaotic attractor, we use two of the more commonly employed methods, nancly phase portraits and power spectrum. The phase plots were obtained integrating equalions (1)-(3) and plotting $X(t)$ vs. $Z(t)$, after letting transients relax (the parameters used are $a=1 / 4, b=1,(i=1)$. The power spectrum was calculated using 4096 points corresponding to the time series of the varialsle $X^{\prime}(t)$ with a time difference $\Delta t=0.5$.

Let us begin by comparing the phase portraits. While the orbits of the attractors $B$ (Fig.la) and $N$ (Fig.1c) look like "noisy limit cycles", the orbit of the strongly chaotic atlractor (F:g.le) appears to fill out a continuous region of the phase space and has a considerably more complicated structure. This impression is reafirmed by the examination of the power spectrum. 'The spectrum of attractors $B$ (Fig.1b) and $N$ (Fig.ld) is composed of broadband components and sharp peaks. In contirast, in the spectrum of the strongly chaotic attractor (Fig.10) we do not distinguish any sharp peaks. These results agree with the fact that attractors $B$ and $N$ arise from period multiplication cascades 

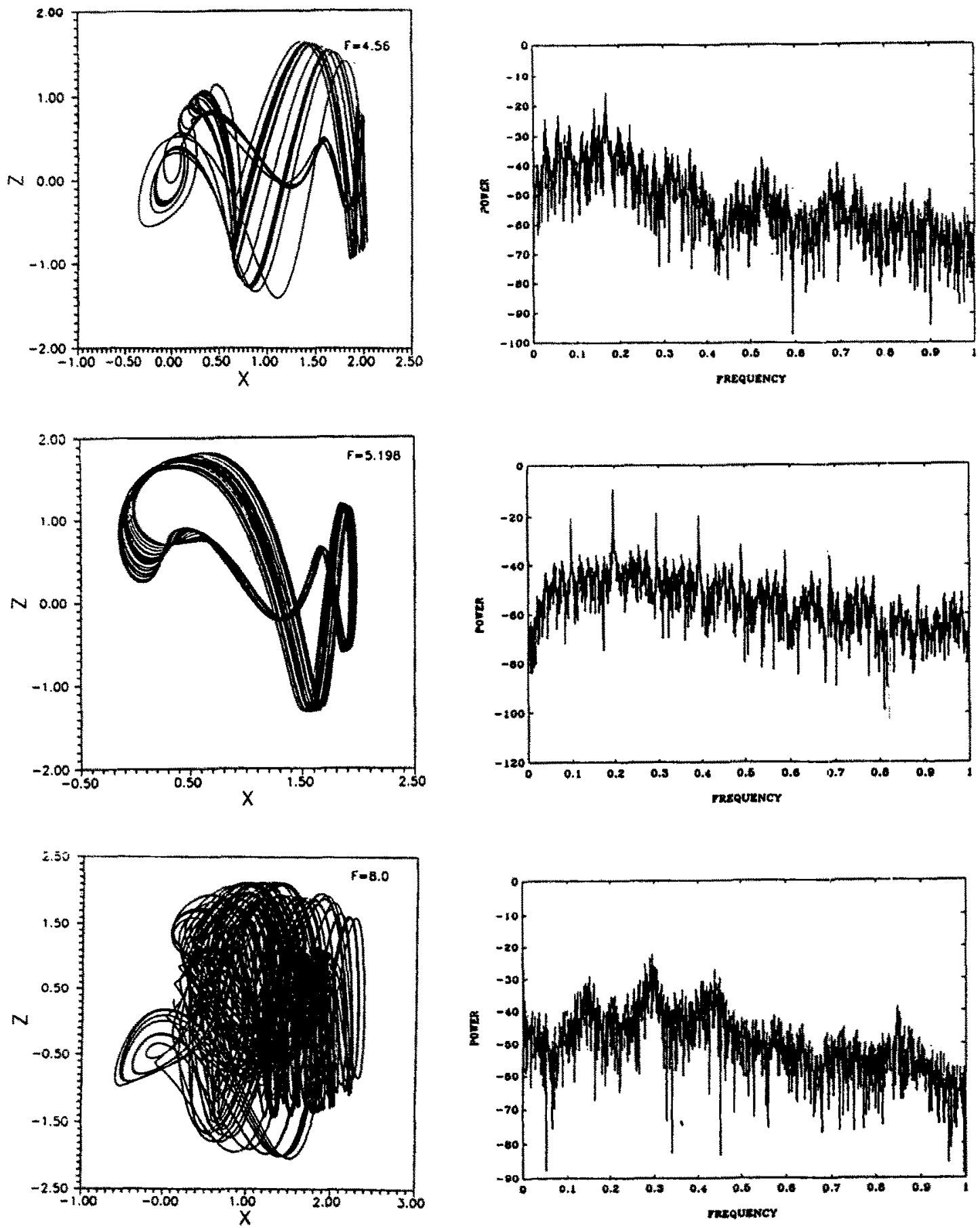

Fig. I Phase plots and power spectra of attractors $B, N$ and turbulent attractor. Parameters: $a=1 / 4, b=4, G=1$ (a) and (b) $F=4.56$; (c) and (d) $F=5.198 ;(e)$ and (f) $F=8.0$. 
of strong limit cycles, while the strongly chaotic attractor is a long chaotic transient that becomes stable when the weak limit cycle losses stability. The lransition scenario of attractor $N$ is a period doubling cascade that will be studied in section 4 , and closer examination of Fig. 1d reveals that the spectrum of attractor $N$ presents sharp peaks at frequencies $n \int_{0}$ with $f_{0} \approx 0.18$ in the frequency of cycle $N$.

\section{POINCARE SECTION ANALYSIS}

In order to gain insight into the geometrical structure of the attractors, we use the Poincare section technique [8]. The Poincare section is a two-dimensional intersection of the phase space chosen in such a way that all qualitatively interesting trajectories actually intersect the plane traniversely. In our case we chose the plane $Z=0$ and plot the coordinates $X$ vs. $Y$ whenever $Z=0$ and $d Z / d !>0$ ("mirror" points are rejected). For the precise location of the intersection point an effective technique proposed by Hénon was employed [19].

Figure 2a shows the Poincaré section of attractor $B$. Five strips can be seen in the figure. Figs. $2 \mathrm{~b}, 2 \mathrm{c}$ and $2 \mathrm{~d}$ are blowups of sectors of the section at successively greater resolution. This attractor shows the recursive structure that occurs on fractal altractors [9]. A careful observation of Fig.3d suggests that there are sectors of the map slightly more probable that others, and that this attractor is a fractal [14].
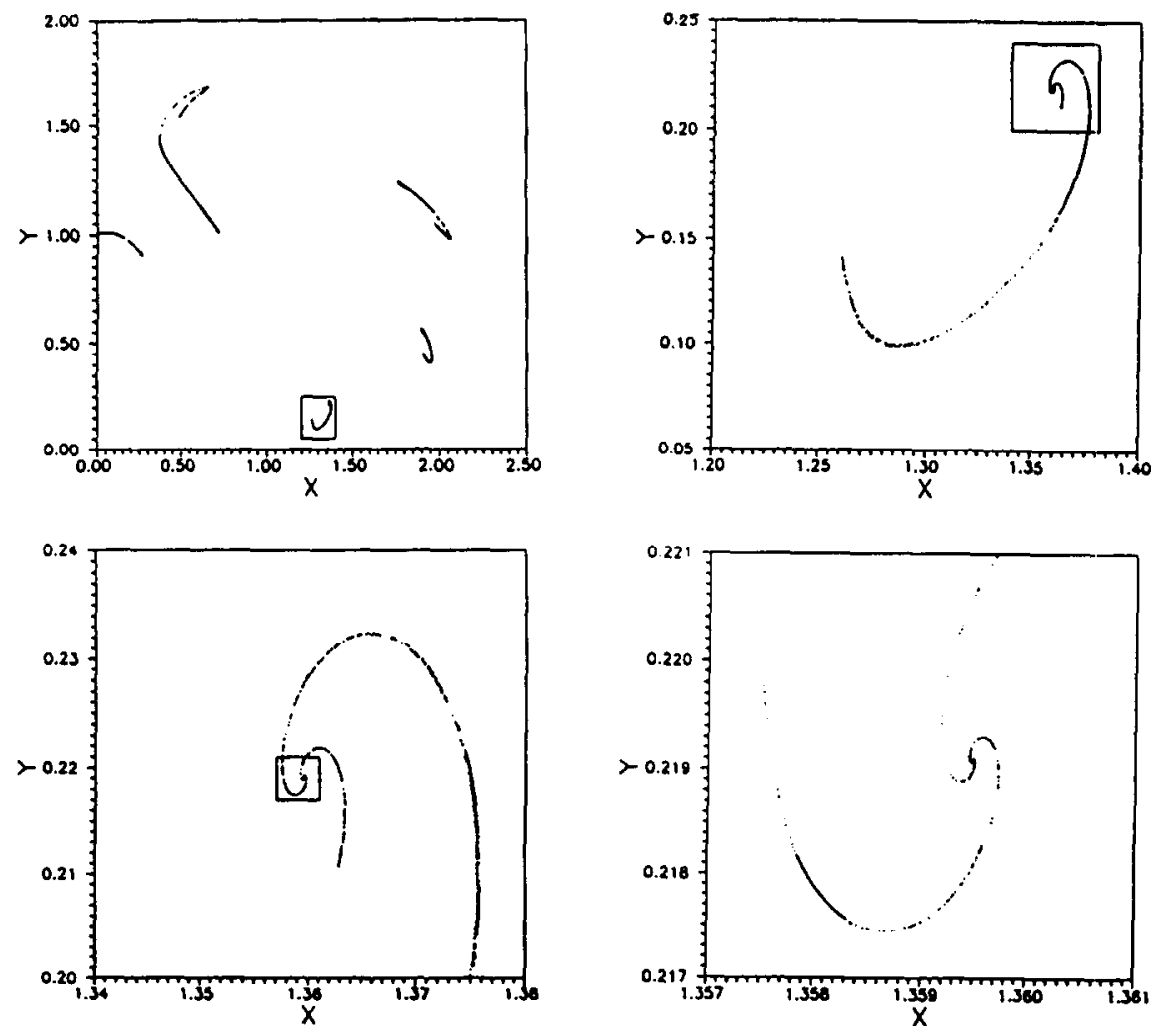

Fig. 2 (a) Poincaré section of attractor $B(F=4.56)$. Figures (b)-(d) are blowups constructed by plotting only those points that lie within the box indicated in figs. (a)-(c). Fig. 2 a contains 5000 points, while (b)-(d) were constructed from 400.000. 


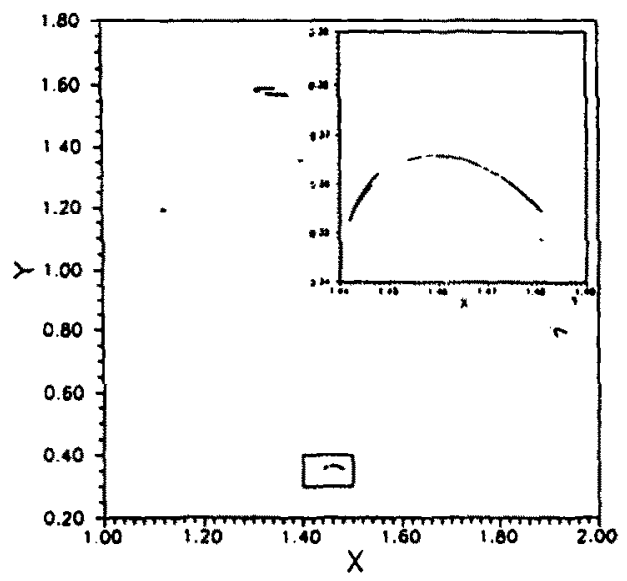

Fig. 3 (a) Poincart section of attractor $N(F=5.198)$. (b) Blowup of the box indicated in fig. $3 a$

Figure 3a shows the Poincare section of allractor $N$, atul Fig. $3 \mathrm{~b}$ is a magnification of the sector indicated in Fig.3a. We see that this attractor consists of three distinct arcs and has simpler structure than the attractor $B$. A series of one-dincusional maljs derived in the next section, from this Poincare map will add us in explaining the secquence of bifurcations observed.

Figure $4 \mathrm{a}$ shows the Poincaré section of the strongly chaotic attractor, and Figs. $4 \mathrm{~b}, 4 \mathrm{c}$ ant $4 \mathrm{~d}$ are successively magnification at greater resolution of a sector of the map. This attractor has pronounced sheet-like Cantor set structure characteristic of chaotic attractors.

\section{PERIOD-DOUBLING ROUTES TO CHAOS}

The routes into chaotic dynamics are studied by means of the Poincare maps defined in the previous section. In $[6,7]$ a sequence of saddle-node and inverse saddle node bifurcations, giving rise to a periodic window structure, was found.

Each periodic window is the domain of a different strong linit cycle and the transition scenario is usually a subharmonic cascade. In this section we will describe three of the period-doubling cascades found in the first part of this series and show that the essential dynamics can be captured by a one-dimensional non-invertible map.

In the first window of periodicity, cycle $B$ period-doubles al $F=4.484$ and follows the subharmonic cascade shown in the bifurcation diagram of figure $5 \mathrm{a}$. This bifurcation diagram was obtained plotting the coordinate $X^{\prime}$ of points in a sector of thel'oincare map, as a function of $F$. We observe that the range of thePoincaré section that is visited by the chaotic trajectory grows with increasing the parameter $\boldsymbol{F}$. Before the end of the Feigenbaum sequence we observe a slarp interior crisis at $F=4.488$ and the size of the chaolic attractor suddenly grows. Beyond this value of $F$ we observe a broad continuation of the chaotic regime up to another crisis at $F=4.489$ that leads to a sudden destruction of the attractor.

In the second window of periodicity, cycle $K$ period-doubles at $F=4.601$ and follows a perioddoubling cascade that is interrupted by a period- halving cascalc.

The corresponding bifurcation diagram is shown in lig. 5b. In the third window cycle $N$ period doubles at $F=5.060^{2}$ and follows the period doubling cascade illustrated in Fig. $5 c$. Before the end of the Feigenbaum sequence, a crisis occurs al $F=5.089$ and leads to a sudden destruction of the chaotic attractor. 

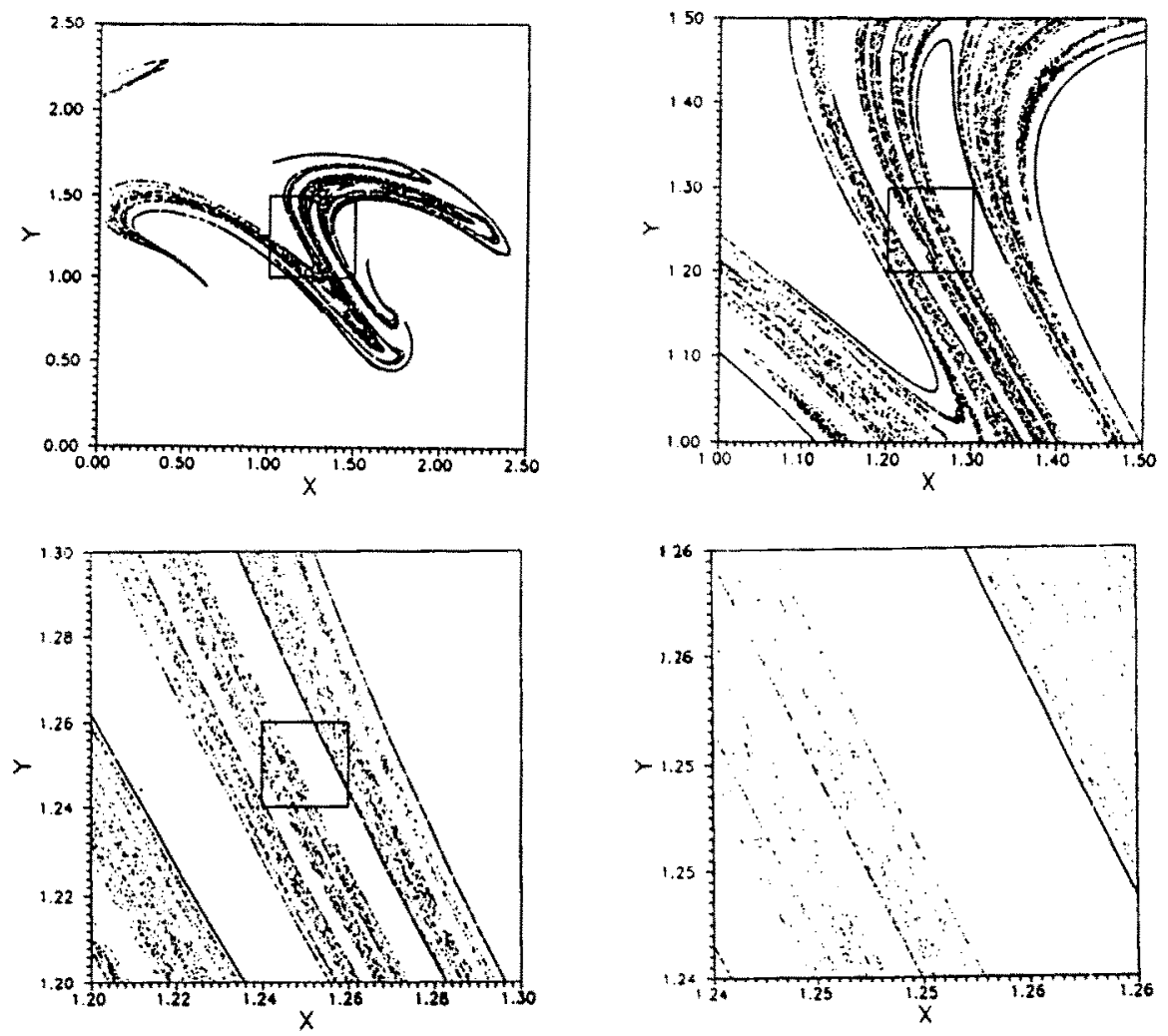

Fig. 4 Poincaré section of the turbulent attractor. The blowups shown in figs (b)-(d) are constructed by plotting only those poin that lie within the box indicated in figs. (a)-(c). Figure 4 a contains only 10000 points, while (b), (c) and (d) were cortstructed fro $1.000,000$ points.
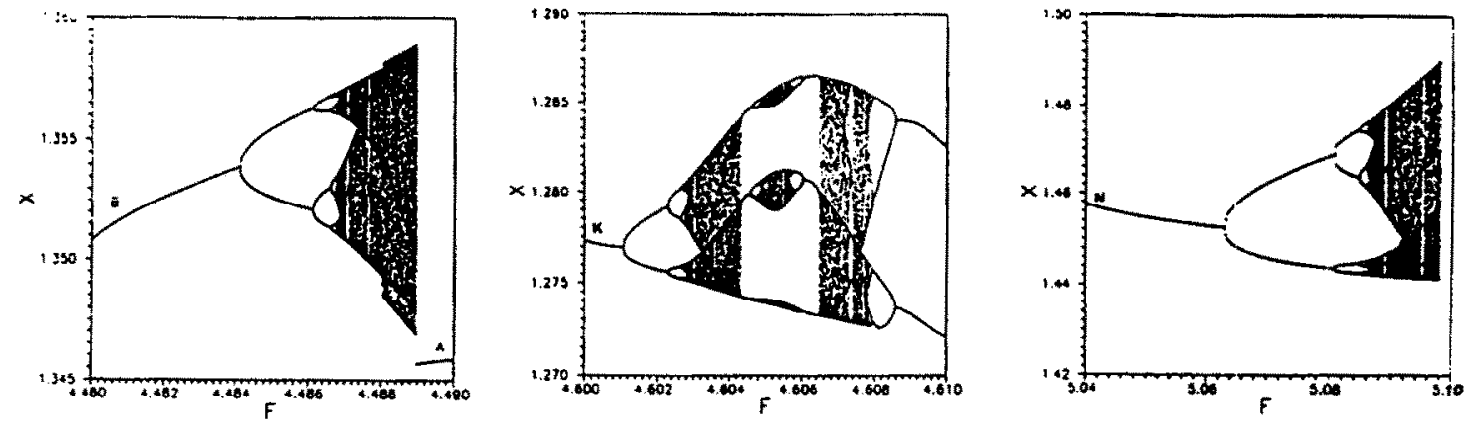

Fig. 5 (a) Bifurcation diagram of cycle B; (b) Bifurcation diagram of cycle K.; (c) Bifurcation diagran of cycle N. 

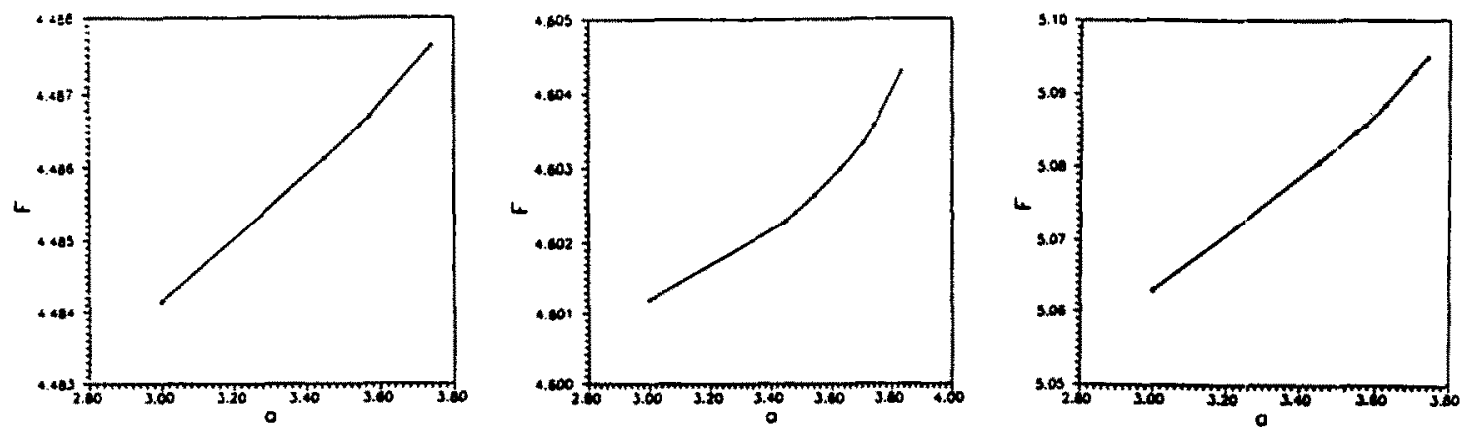

Fig. 6 Relation between the critical values of the parameter $a$ in the Feigenbaum's sequence of bifurcations and the values of the parameter $F$ at which the analogous events occur in the dynamics of the model. a) Bifurcations of cycle $B$. b) Bifurcations of cyc K. c) Bifurcations of cycle $\mathrm{N}$.

The first part of the three bifurcation diagrams resembles the structure of the attractors of the logistic map on the unit interval $X_{n+1}=a X_{n}\left(1-X_{n}\right)[17,18]$. Morcover, there is an almost linear relation between the values of the parameter $a$ and the paraneter $F$ at which the period doublings and periodic windows in the chaotic regine occur (see Fig.6). The sequence of events leading into chaos and the fine structure of the cliaotic regime along these routes show perfect correnpondence to the Feigenbaum or Sharbovski sequence.

For few selected values of $F$ in the bifurcation cascade of cycle $N$ we constructed the first return maps on the unit interval, shown in Fig.7. 'I'hese maps were constructed from points in the sector
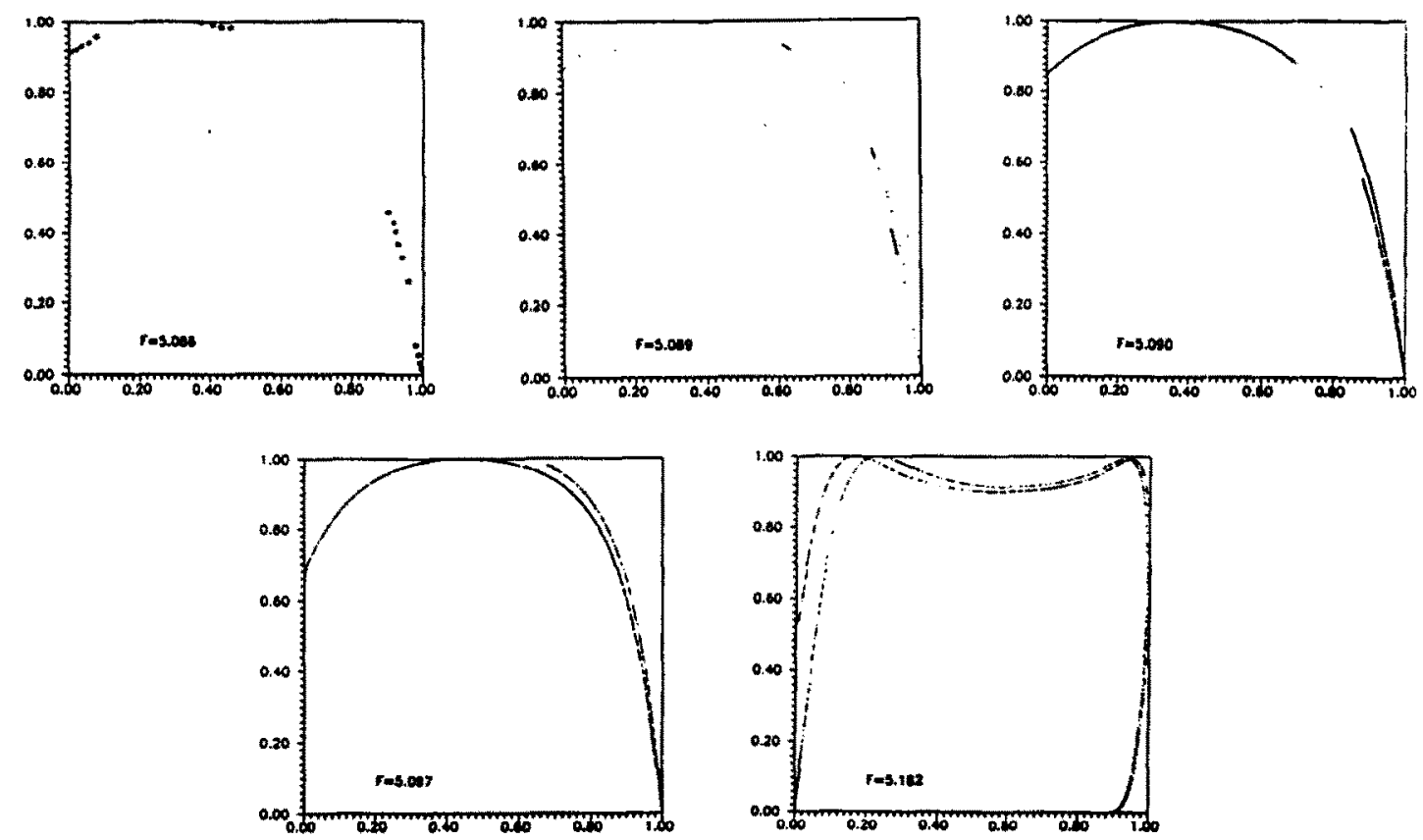

Fig. 7 Return maps $\left(w_{n}, w_{n+1}\right)$ on the unit interval corresponding to the period-doubling cascade of cycle $B$. The parameters are (a) $F=5.086,(b) F=5.089$, (c) $F=5.09$, (d) $F=5.097$, (c) $F=5.182$ The points $w=0$ and $w=1$ correspond to the outermost and innermost points of the sector of the Poincare section indicated in fig. 4. 
of the Poincare map indicated in Fig.3b. The coordinate $X$ of the outermost point of the sector was defined as $\omega=0$ and the coordinate $X$ of the immomost point was chosen to represent the value $\omega=1$. As expected, the maps resemble the logistic map in the parameter range where we observe the Feigenbaum sequence. However, at higher valtes of the may becomes more complicated.

\section{CHARACTERIZATION OF THE ATTRACTORS}

Lyapunov exponents describe the mean mate of expenkntial divergence or convergence of initially neighboring trajectories and provide the best quamtitive measure of chaotic behavior. A system with one or more positive Lyapunov exponents is delined to be chaotic. We have calculated the Lyapunov exponents using the method proposed by Woll ct. al.[10] since this techniqueyields in a straightforward manner for computing the complete Lyapunov spectrum. 'The results obtained were double-checked by applying alternative methods such as those given by Benettin et. al. [11] and Eckmann et. al.[12].

Table I summarize the results obtained. The Lyapunov calculations clearly indicate chaotic behavior, since all the attractors have one positive Lyaptuov exponent. Nevertheless, the strongly chaotic attractor presents a considerable larger chaotic dymamics than attractors $B$ and $N$, since its positive exponent is larger than the positive exponent of attractors $B$ and $N$ and its negative exponent is smaller than the negative exponent of attractors $B$ and $N$.

In order to study the fractal nature of the attractors quanlitatively, 2we now turn to the calculation of the dimension of the attractors. The methoul proposed by Grassberger and Procaccia $[14,15]$ leads the determination of the correlation timension $D_{i}$ which is a lower bound on the Hausdorff dimension. The correlation sum:

$$
C(r)=\frac{1}{N^{2}}\left[N \text { umberofpairs }\left(x_{i}, x_{j}\right) \text { with }\left|x_{i}-x_{j}\right|<r\right]
$$

was calculated and the scaling region in $\mathrm{r}$ for which $C(r) \approx r^{*}$ was located. If $\mu$ approaches a limiting value $D_{c}$ as the embeding dimension is increased, $D_{c}$ is identifed as the correlation dimension. The correlation dimension was calculated from the linree dincusional time series $x_{i}(t), y_{i}(t), z_{i}(t)$ as well as from the reconstructed series $x\left(t_{i}\right), x\left(t_{i}+\tau\right), x\left(t_{i}+2 \tau\right)$, applyiug the delay method to reconstruct the dynamics from the measurements of a single variable. In both cases 70.000 data points were used, and for the reconstruction of the attractor, a delay time $T=1$ was chosen. The estimated correlation dimensions appear in Table 1 . We observe a very good agreement butween both calculations. While attractors $B$ and $N$ present a correlation dimension $1<D_{c}<2$, according with the topological shape of the attractors ("noisy limil cycles"), the strougly chaotic attractor has a correlation dimension $D_{c}>2$ evidencing a more complex dyuamics. In addition, the Lyapunov dimension $D_{L}$ was calculated from the Kaplan and Yorke's formula

$$
D_{L}=j+\frac{\sum_{i=1}^{j} \lambda_{i}}{\left|\lambda_{j+1}\right|}
$$

where $j$ is the largest integer for which $\lambda_{1}+\ldots+\lambda_{j} \geq 0$. For typical attractors it has been conjectured [16] that the Lyapunov dimension is equal to the information dimension and thus gives a value $D_{L} \geq D_{c}$. Our results are given in Table!.

TABLE I Lyapunov exponents and dimensions for attractors $B, N$, and strongly chactic

\begin{tabular}{|l|lll|l|l|l|}
\hline \multicolumn{1}{|c|}{ Attractor } & \multicolumn{3}{|c|}{ Lyapunov Exponents } & $D_{L}$ & $D_{c}(1)$ & $D_{c}(2)$ \\
\hline $\mathrm{B}(\mathrm{F}=4.56)$ & 0.07 & 0 & 1.0 & 2.07 & 1.50 & 1.48 \\
$\mathrm{~N}(\mathrm{~F}=5.198)$ & 0.07 & 0 & 0.85 & 2.08 & 1.79 & 1.78 \\
Strongly chaotic $(\mathrm{F}=8.0)$ & 0.23 & 0 & -0.50 & 2.39 & 2.23 & 2.22 \\
\hline
\end{tabular}

(1) Calculated from the reconstructed time series $x\left(t_{i}\right), x\left(t_{i}+\tau\right), x\left(t_{i}+2 \tau\right)$ with $\tau=1$.

(2) Calculated from the three dimensional time series $x_{i}(t), y_{i}(t), z_{i}(t)$ 


\section{DISCUSSION}

In this contribution we have presented a full characterization of the chaotic attractors of the Lorenz model of general circulation of the atmosphere.

Direct evidence from the inspection of computed trajectories shows that attractors $B$ and $N$ look like "noisy limit cycles", while the strongly chatic attractor presents a much more complicated structure. In addition the spectra of attractors $B$ and $N$ present sharp peaks and broad-band components. On the contrary, in the spectrum of the strongly chaotic attractor we discern no sharp peaks. These features confirm the results of ref. [7] while the transition scenarios of attractors $B$ and $N$ are period-multiplication cascades, the transition scenario of the strongly chaotic attractor is a subcritical Hopf bifurcation of the weak limit cycle.

Moreover, the Poincare sections of attractors $B$ and $N$ consist of thin arcs while the Poincaré section of the strongly chaotic attractor presents a complicated self similar sheet-like structure. With the aid of Poincare sections the period doubling cascades were studied in detail. We were able to show that chaos develops in an almost perlect Peigenbaum scenario. However, the chaos in never fully developed, but it ends with a crisis or a period-halving cascade. The detailed study of these crises is the object of a forthcoming paper.

Dimension and Lyapunov exponent calculations confirm the fractal and chaotic nature of the attractors. The Lyapunov exponents and dimensions of attractors $B$ and $N$ are quantitalively different from those of the strongly chaotic attractor. The values are lower, indicating much thinner strange attractors.

Acknowledgment This work was supported in part by the "Proyecto Tecnológico CONICYT-BID" of the Consejo Nacional de Investigaciones Cientificas y Técnicas (CONICYT, URUGUAY); by PEDECIBA (Project URU/84/002 - UNDP) and Comisión Sectorial de lıvestigación Científica (CSIC, URUGUAY) and by PID 3-00571/88 (CONICET,ARGEN'TINA)

\section{REFERENCES}

1. H.G. Schuster, Deterministic Chaos, An lntroduction 2nd. Ed. (VCH, Weinheim, 1988).

2. Hao Bai-Lin, Elementary Symbolic Dynamics and Claas in Dissipative Systems (World Scientific, Singapore, 1989).

3. E. N. Lorenz, Tellus 36A (1984) 98.

4. E. N. Lorenz, Tellus 42A (1990) 378.

5. E. N. Lorenz, J. Atmos. Sci. 20 (1963) 130.

6. C. Masoller, A. Sicardi and L. Romanelli, Physics Letters A 167 (1992) 185.

7. C. Masoller, A. Sicardi Schifino and L. Romanclli, submitted to Journal of the Atmospheric Sciences

8. P. Bergé, Y. Pomeau and C. Vidal, Order Within ('haos (Wiley, 1986).

9. B.B. Mandelbrot, The Fractal Geometry of Nature (W.H. Freeman, New York, 1983)

10. A. Wolf, J.B. Swift, H.L. Swinney and A. Vastano, Physica D 16 (1985) 285.

11. G. Bennetin, L. Galgani, A. Giorgilli and J. M. Strelcyu, Meccanica 15 (1980) 9. 
12. J-P. Eckmann, S.O. Kamphorst, D. Ruelle and S. Ciliberto, Phys. Rev. A 34 (1986) 4971.

13. J.D. Farmer, E. Ott, J.A. Yorke, Physica D 7 (1983) 153

14. P. Grassberger, I. Procaccia, Physica D 9 (1983) 189.

15. P. Grassberger and I. Procaccia, Phys. Rev. Lett. 50 (1983) 346.

16. J.L. Kaplan and J.A. Yorke, Lecture Notes in Mallenatics 730 (Springer- Verlag, 1979) p. 228; P. Frederickson, J. Kaplan, E. Yorke and J. Yorke, J.Dilf. Eqs. 49 (1983) 185.

17. M.J. Feigenbaum, J. Stat. Phys. 19 (1978) 25; J. Stat. Phys. 21 (1979) 669.

18. P. Collet and J-P Eckmann, Iterated Maps in the lnterval as Dynamical Systems (Birkhituser, Boston, 1980).

19. M. Hénon, Physica D 5 (1983) 412. 